\title{
Focal representation of k-slant Helices in $\mathbb{E}^{\mathrm{m}+1}$
}

\author{
Günay Öztürk \\ Department of Mathematics \\ Kocaeli University, Turkey \\ email: ogunay@kocaeli.edu.tr \\ Bengü Bayram \\ Department of Mathematics \\ Balıkesir University, Turkey \\ email: benguk@balikesir.edu.tr
}

\author{
Betül Bulca \\ Department of Mathematics \\ Uludağ University, Turkey \\ email: bbulca@uludag.edu.tr \\ Kadri Arslan \\ Department of Mathematics \\ Uludağ University, Turkey \\ email: arslan@uludag.edu.tr
}

\begin{abstract}
The focal representation of a generic regular curve $\gamma$ in $\mathbb{E}^{m+1}$ consists of the centers of the osculating hyperplanes. A k-slant helix $\gamma$ in $\mathbb{E}^{\mathrm{m}+1}$ is a (generic) regular curve whose unit normal vector $\mathrm{V}_{\mathrm{k}}$ makes a constant angle with a fixed direction $\vec{U}$ in $\mathbb{E}^{m+1}$. In the present paper we proved that if $\gamma$ is a $k$-slant helix in $\mathbb{E}^{\mathrm{m}+1}$, then the focal representation $\mathrm{C}_{\gamma}$ of $\gamma$ in $\mathbb{E}^{\mathrm{m}+1}$ is an $(\mathrm{m}-\mathrm{k}+2)$-slant helix in $\mathbb{E}^{\mathrm{m+1}}$.
\end{abstract}

\section{Introduction}

Curves with constant slope, or so-called general helices (inclined curves), are well-known curves in the classical differential geometry of space curves. They are defined by the property that the tangent makes a constant angle with a fixed line (the axis of the general helix) (see, [1], [4], [7] and [8]). In [10], the definition is more restrictive: the fixed direction makes constant angle with these all the vectors of the Frenet frame. It is easy to check that the definition 
only works in the odd dimensional case. Moreover, in the same reference, it is proven that the definition is equivalent to the fact the ratios $\frac{K_{2}}{k_{1}}, \frac{K_{4}}{k_{3}}, \ldots$, $\kappa_{i}$ being the curvatures, are constant. Further, J. Monterde has considered the Frenet curves in $\mathbb{E}^{m}$ which have constant curvature ratios (i.e., $\frac{k_{2}}{k_{1}}, \frac{k_{3}}{k_{2}}, \frac{k_{4}}{k_{3}} \ldots$ are constant) [14]. The Frenet curves with constant curvature ratios are called ccr-curves. Obviously, ccr-curves are a subset of generalized helices in the sense of [10]. It is well known that curves with constant curvatures (W-curves) are well-known ccr-curves [12], [15].

Recently, Izumiya and Takeuchi have introduced the concept of slant helix in Euclidean 3-space $\mathbb{E}^{3}$ by requiring that the normal lines make a constant angle with a fixed direction [11]. Further in [3] Ali and Turgut considered the generalization of the concept of slant helix to Euclidean $n$-space $\mathbb{E}^{n}$, and gave some characterizations for a non-degenerate slant helix. As a future work they remarked that it is possible to define a slant helix of type-k as a curve whose unit normal vector $V_{k}$ makes a constant angle with a fixed direction $\vec{u}$ [9]

For a smooth curve (a source of light) $\gamma$ in $\mathbb{E}^{\mathrm{m}+1}$, the caustic of $\gamma$ (defined as the envelope of the normal lines of $\gamma$ ) is a singular and stratified hypersurface. The focal curve of $\gamma, C_{\gamma}$, is defined as the singular stratum of dimension 1 of the caustic and it consists of the centers of the osculating hyperspheres of $\gamma$. Since the center of any hypersphere tangent to $\gamma$ at a point lies on the normal plane to $\gamma$ at that point, the focal curve of $\gamma$ may be parametrized using the Frenet frame $\left(t, n_{1}, n_{2}\right.$, dots, $\left.n_{m}\right)$ of $\gamma$ as follows:

$$
C_{\gamma}(\theta)=\left(\gamma+c_{1} n_{1}+c_{2} n_{2}+\cdots+c_{m} n_{m}\right)(\theta),
$$

where the coefficients $c_{1}, \ldots, c_{m}$ are smooth functions that are called focal curvatures of $\gamma[18]$.

This paper is organized as follows: Section 2 gives some basic concepts of the Frenet curves in $\mathbb{E}^{\mathrm{m}+1}$. Section 3 tells about the focal representation of a generic curve given with a regular parametrization in $\mathbb{E}^{m+1}$. Further this section provides some basic properties of focal curves in $\mathbb{E}^{\mathfrak{m}+1}$ and the structure of their curvatures. In the final section we consider k-slant helices in $\mathbb{E}^{\mathrm{m}+1}$. We prove that if $\gamma$ is a $k$-slant helix in $\mathbb{E}^{m+1}$ then the focal representation $C_{\gamma}$ of $\gamma$ is an $(m-k+2)$-slant helix in $\mathbb{E}^{m+1}$.

\section{Basic concepts}

Let $\gamma=\gamma(\mathrm{s}): \mathrm{I} \rightarrow \mathbb{E}^{\mathrm{m}+1}$ be a regular curve in $\mathbb{E}^{\mathrm{m}+1}$, (i.e., $\left\|\gamma^{\prime}(\mathrm{s})\right\|$ is nowhere zero) where I is an interval in $\mathbb{R}$. Then $\gamma$ is called a Frenet curve of osculating 
order $\mathrm{d},(2 \leq \mathrm{d} \leq \mathrm{m}+1)$ if $\gamma^{\prime}(\mathrm{s}), \gamma^{\prime \prime}(\mathrm{s}), \ldots, \gamma^{(\mathrm{d})}(\mathrm{s})$ are linearly independent and $\gamma^{\prime}(s), \gamma^{\prime \prime}(s), \ldots, \gamma^{(d+1)}(s)$ are no longer linearly independent for all $s$ in I [18]. In this case, $\operatorname{Im}(\gamma)$ lies in a d-dimensional Euclidean subspace of $\mathbb{E}^{m+1}$. To each Frenet curve of rank $d$ there can be associated the orthonormal $\mathrm{d}$-frame $\left\{t, n_{1}, \ldots, n_{d-1}\right\}$ along $\gamma$, the Frenet $r$-frame, and $d-1$ functions $\kappa_{1}, \kappa_{2}, \ldots, \kappa_{d-1}: I \longrightarrow \mathbb{R}$, the Frenet curvatures, such that

$$
\left[\begin{array}{c}
\mathrm{t}^{\prime} \\
\mathrm{n}_{1}^{\prime} \\
\mathrm{n}_{2}^{\prime} \\
\cdots \\
\mathrm{n}_{\mathrm{d}-1}^{\prime}
\end{array}\right]=v\left[\begin{array}{ccccc}
0 & \mathrm{k}_{1} & 0 & \cdots & 0 \\
-\mathrm{k}_{1} & 0 & \mathrm{k}_{2} & \cdots & 0 \\
0 & -\mathrm{k}_{2} & 0 & \cdots & 0 \\
\cdots & & & & \mathrm{k}_{\mathrm{d}-1} \\
0 & 0 & \cdots & -\mathrm{k}_{\mathrm{d}-1} & 0
\end{array}\right]\left[\begin{array}{c}
\mathrm{t} \\
\mathrm{n}_{1} \\
\mathrm{n}_{2} \\
\cdots \\
\mathrm{n}_{\mathrm{d}-1}
\end{array}\right]
$$

where, $v$ is the speed of $\gamma$. In fact, to obtain $t, n_{1}, \ldots, n_{d-1}$ it is sufficient to apply the Gram-Schmidt orthonormalization process to $\gamma^{\prime}(\mathrm{s}), \gamma^{\prime \prime}(\mathrm{s}), \ldots, \gamma^{(\mathrm{d})}(\mathrm{s})$. Moreover, the functions $\mathrm{K}_{1}, \mathrm{~K}_{2}, \ldots, \mathrm{K}_{\mathrm{d}-1}$ are easily obtained as by-product during this calculation. More precisely, $t, n_{1}, \ldots, n_{d-1}$ and $\kappa_{1}, \kappa_{2}, \ldots, \kappa_{d-1}$ are determined by the following formulas:

$$
\begin{aligned}
v_{1}(s): & =\gamma^{\prime}(s) \quad ; t:=\frac{v_{1}(s)}{\left\|v_{1}(s)\right\|}, \\
v_{\alpha}(s): & =\gamma^{(\alpha)}(s)-\sum_{i=1}^{\alpha-1}<\gamma^{(\alpha)}(s), v_{i}(s)>\frac{v_{i}(s)}{\left\|v_{i}(s)\right\|^{2}}, \\
\kappa_{\alpha-1}(s): & =\frac{\left\|v_{\alpha}(s)\right\|}{\left\|v_{\alpha-1}(s)\right\|\left\|v_{1}(s)\right\|}, \\
n_{\alpha-1}: & =\frac{v_{\alpha}(s)}{\left\|v_{\alpha}(s)\right\|},
\end{aligned}
$$

where $\alpha \in\{2,3, \ldots, d\}$ (see, $[8]$ ).

A Frenet curve of rank $d$ for which $\kappa_{1}, \kappa_{2}, \ldots, \kappa_{d-1}$ are constant is called (generalized) screw line or helix [6]. Since these curves are trajectories of the 1-parameter group of the Euclidean transformations, so, F. Klein and S. Lie called them $W$-curves [12]. For more details see also [5]. $\gamma$ is said to have constant curvature ratios (that is to say, it is a ccr-curve) if all the quotients $\frac{k_{2}}{k_{1}}, \frac{k_{3}}{k_{2}}, \frac{k_{4}}{k_{3}}, \ldots, \frac{k_{i}}{k_{i-1}}(1 \leq i \leq m-1)$ are constant [14], [15].

\section{The focal representation of a curve in $\mathbb{E}^{m+1}$}

The hyperplane normal to $\gamma$ at a point is the union of all lines normal to $\gamma$ at that point. The envelope of all hyperplanes normal to $\gamma$ is thus a component 
of the focal set that we call the main component (the other component is the curve $\gamma$ itself, but we will not consider it) [16].

Definition 1 Given a generic curve (i.e., a Frenet curve of osculating order $\mathrm{m}+1) \gamma: \mathbb{R} \rightarrow \mathbb{E}^{\mathrm{m}+1}$, let $\mathrm{F}: \mathbb{E}^{\mathrm{m}+1} \times \mathbb{R} \rightarrow \mathbb{R}$ be the $(\mathrm{m}+1)$-parameter family of real functions given by

$$
F(q, \theta)=\frac{1}{2}\|q-\gamma(\theta)\|^{2}
$$

The caustic of the family $\mathrm{F}$ is given by the set

$$
\left\{\mathrm{q} \in \mathbb{E}^{\mathrm{m}+1}: \exists \theta \in \mathbb{R}: \mathrm{F}_{\mathrm{q}}^{\prime}(\theta)=0 \text { and } \mathrm{F}_{\mathrm{q}}^{\prime \prime}(\theta)=0\right\}
$$

[16].

Proposition 1 [17] The caustic of the family $\mathrm{F}(\mathrm{q}, \theta)=\frac{1}{2}\|\mathrm{q}-\gamma(\theta)\|^{2}$ coincides with the focal set of the curve $\gamma: \mathbb{R} \rightarrow \mathbb{E}^{\mathrm{m}+1}$.

Definition 2 The center of the osculating hypersphere of $\gamma$ at a point lies in the hyperplane normal to the $\gamma$ at that point. So we can write

$$
C_{\gamma}=\gamma+c_{1} n_{1}+c_{2} n_{2}+\cdots+c_{m} n_{m}
$$

which is called the focal curve of $\gamma$, where $\mathrm{c}_{1}, \mathrm{c}_{2}, \ldots, \mathrm{c}_{\mathrm{m}}$ are smooth functions of the parameter of the curve $\gamma$. We call the function $\boldsymbol{c}_{i}$ the $i^{\text {th }}$ focal curvature of $\gamma$. Moreover, the function $\mathrm{c}_{1}$ never vanishes and $\mathrm{c}_{1}=\frac{1}{\mathrm{k}_{1}}$ [18].

The focal curvatures of $\gamma$, parametrized by arc length s, satisfy the following "scalar Frenet equations" for $c_{m} \neq 0$ :

$$
\begin{aligned}
1= & \mathrm{K}_{1} \mathrm{c}_{1} \\
\mathrm{c}_{1}= & \mathrm{\kappa}_{2} \mathrm{c}_{2} \\
\mathrm{c}_{2}= & -\mathrm{K}_{2} \mathrm{c}_{1}+\mathrm{K}_{3} \mathrm{c}_{3} \\
& \cdots \\
\mathrm{c}_{\mathrm{m}-1}= & -\mathrm{\kappa}_{\mathrm{m}-1} \mathrm{c}_{\mathrm{m}-2}+\mathrm{K}_{\mathrm{m}} \mathrm{c}_{\mathrm{m}} \\
\mathrm{c}_{\mathrm{m}}-\frac{\left(\mathrm{R}_{\mathrm{m}}^{2}\right)}{2 \mathrm{c}_{\mathrm{m}}}= & -\mathrm{K}_{\mathrm{m}} \mathrm{c}_{\mathrm{m}-1}
\end{aligned}
$$

where $R_{m}$ is the radius of the osculating $m$-sphere. In particular $R_{m}^{2}=\left\|C_{\gamma}-\gamma\right\|^{2}$ [18]. 
Theorem 1 [16] Let $\gamma: \mathrm{s} \rightarrow \gamma(\mathrm{s}) \in \mathbb{E}^{\mathrm{m}+1}$ be a regular generic curve. Write $\mathrm{K}_{1}, \mathrm{~K}_{2}, \ldots, \mathrm{K}_{\mathrm{m}}$ for its Euclidean curvatures and $\left\{\mathrm{t}, \mathrm{n}_{1}, \mathrm{n}_{2}, \ldots, \mathrm{n}_{\mathrm{m}}\right\}$ for its Frenet Frame. For each non-vertex $\gamma(\mathrm{s})$ of $\gamma$, write $\varepsilon(\mathrm{s})$ for the sign of $\left(\mathrm{c}_{\mathrm{m}}^{\prime}+\mathrm{c}_{\mathrm{m}-1} \mathrm{~K}_{\mathrm{m}}\right)(\mathrm{s})$ and $\delta_{\alpha}(\mathrm{s})$ for the sign of $(-1)^{\alpha} \mathcal{\varepsilon}(\mathrm{s}) \mathrm{\kappa}_{\mathrm{m}}(\mathrm{s}), \alpha=1, \ldots, \mathrm{m}$. Then the following holds:

a) The Frenet frame $\left\{\mathrm{T}, \mathrm{N}_{1}, \mathrm{~N}_{2}, \ldots, \mathrm{N}_{\mathrm{m}}\right\}$ of $\mathrm{C}_{\gamma}$ at $\mathrm{C}_{\gamma}(\mathrm{s})$ is well-defined and its vectors are given by $\mathrm{T}=\varepsilon_{\mathrm{m}}, \mathrm{N}_{\alpha}=\delta_{\alpha} \mathrm{n}_{\mathrm{m}-\mathrm{l}}$, for $\mathrm{l}=1, \ldots, \mathrm{m}-1$, and $\mathrm{N}_{\mathrm{m}}= \pm \mathrm{t}$. The sign in $\pm \mathrm{t}$ is chosen in order to obtain a positive basis.

b) The Euclidean curvatures $\mathrm{K}_{1}, \mathrm{~K}_{2}, \ldots, \mathrm{K}_{\mathrm{m}}$ of the parametrized focal curve of $\gamma, \mathrm{C}_{\gamma}: \mathrm{s} \rightarrow \mathrm{C}_{\gamma}(\mathrm{s})$, are related to those of $\gamma$ by:

$$
\frac{\mathrm{K}_{1}}{\left|\mathrm{~K}_{\mathrm{m}}\right|}=\frac{\mathrm{K}_{2}}{\mathrm{~K}_{\mathrm{m}-1}}=\cdots=\frac{\left|\mathrm{K}_{\mathrm{m}}\right|}{\mathrm{K}_{1}}=\frac{1}{\left|\mathrm{c}_{\mathrm{m}}^{\prime}+\mathrm{c}_{\mathrm{m}-1} \mathrm{~K}_{\mathrm{m}}\right|},
$$

the sign of $\mathrm{K}_{\mathrm{m}}$ is equal to $\delta_{\mathrm{m}}$ times the sign chosen in $\pm \mathrm{t}$.

That is the Frenet formulas of $\mathrm{C}_{\gamma}$ at $\mathrm{C}_{\gamma}(\mathrm{s})$ are

$$
\begin{aligned}
& \mathrm{T}^{\prime}=\frac{1}{\mathrm{~A}}\left|\mathrm{\kappa}_{\mathrm{m}}\right| \mathrm{N}_{1} \\
& N_{1}^{\prime}=\frac{1}{A}\left(-\left|\kappa_{m}\right| T+\kappa_{m-1} N_{2}\right) \\
& N_{2}^{\prime}=\frac{1}{A}\left(-\left|\kappa_{m-1}\right| N_{1}+\kappa_{m-2} N_{3}\right) \\
& \mathrm{N}_{\mathrm{m}-1}^{\prime}=\frac{\ddot{1}}{\mathrm{~A}}\left(-\mathrm{K}_{2} \mathrm{~N}_{\mathrm{m}-2} \mp \delta_{\mathrm{m}} \mathrm{K}_{1} \mathrm{~N}_{\mathrm{m}}\right) \\
& \mathrm{N}_{\mathrm{m}}^{\prime}=\frac{1}{\mathrm{~A}} \mp \delta_{\mathrm{m}} \mathrm{K}_{1} \mathrm{~N}_{\mathrm{m}-1}
\end{aligned}
$$

where $A=\left|c_{m}^{\prime}+c_{m-1} k_{m}\right|$.

Corollary 1 Let $\gamma=\gamma(\mathrm{s})$ be a regular generic curve in $\mathbb{E}^{\mathrm{m}+1}$ and $\mathrm{C}_{\gamma}: \mathrm{s} \rightarrow$ $\mathrm{C}_{\gamma}(\mathrm{s})$ be the focal representation of $\gamma$. Then the Frenet frame of $\mathrm{C}_{\gamma}$ becomes as follows;

i) If $\mathrm{m}$ is even, then

$$
\begin{aligned}
\mathrm{T}= & \mathrm{n}_{\mathrm{m}} \\
\mathrm{N}_{1}= & -\mathrm{n}_{\mathrm{m}-1} \\
\mathrm{~N}_{2}= & \mathrm{n}_{\mathrm{m}-2} \\
& \cdots \\
\mathrm{N}_{\mathrm{m}-1}= & -\mathrm{n}_{1} \\
\mathrm{~N}_{\mathrm{m}}= & \mathrm{t}
\end{aligned}
$$


ii) If $\mathrm{m}$ is odd, then

$$
\begin{aligned}
\mathrm{T} & =\mathrm{n}_{\mathrm{m}} \\
\mathrm{N}_{1}= & -\mathrm{n}_{\mathrm{m}-1} \\
\mathrm{~N}_{2}= & \mathrm{n}_{\mathrm{m}-2} \\
& \cdots \\
\mathrm{N}_{\mathrm{m}-1}= & \mathrm{n}_{1} \\
\mathrm{~N}_{\mathrm{m}}= & -\mathrm{t} .
\end{aligned}
$$

Proof. By the use of (7) with (8) we get the result.

\section{4 k-Slant helices}

Let $\gamma=\gamma(\mathrm{s}): \mathrm{I} \rightarrow \mathbb{E}^{\mathrm{m}+1}$ be a regular generic curve given with arclength parameter. Further, let $\overrightarrow{\mathrm{U}}$ be a unit vector field in $\mathbb{E}^{\mathrm{m}+1}$ such that for each $s \in \mathrm{I}$ the vector $\overrightarrow{\mathrm{U}}$ is expressed as the linear combinations of the orthogonal basis $\left\{V_{1}(s), V_{2}(s), \ldots, V_{m+1}(s)\right\}$ with

$$
\overrightarrow{\mathrm{u}}=\sum_{j=1}^{m+1} a_{j}(s) V_{j}(s) .
$$

where $a_{j}(s)$ are differentiable functions, $1 \leq j \leq m+1$.

Differentiating $\overrightarrow{\mathrm{u}}$ and using the Frenet equations (1), one can get

$$
\frac{d \vec{U}}{d s}=\sum_{i=1}^{m+1} P_{i}(s) V_{i}(s),
$$

where

$$
\begin{aligned}
P_{1}(s) & =a_{1}^{\prime}-\kappa_{1} a_{2}, \\
P_{i}(s) & =a_{i}^{\prime}+\kappa_{i-1} a_{i-1}-\kappa_{i} a_{i+1}, 2 \leq i \leq m, \\
P_{m+1}(s) & =a_{m+1}^{\prime}+\kappa_{m} a_{m} .
\end{aligned}
$$

If the vector field $\overrightarrow{\mathrm{u}}$ is constant then the following system of ordinary differential equations are obtained

$$
\begin{aligned}
& 0=a_{1}^{\prime}-\kappa_{1} a_{2}, \\
& 0=a_{2}^{\prime}+\kappa_{1} a_{1}-\kappa_{2} a_{3}, \\
& 0=a_{i}^{\prime}+\kappa_{i-1} a_{i-1}-\kappa_{i} a_{i+1}, 3 \leq i \leq m, \\
& 0=a_{m+1}^{\prime}+\kappa_{m} a_{m} .
\end{aligned}
$$


Definition 3 Recall that a unit speed generic curve $\gamma=\gamma(\mathrm{s}): \mathrm{I} \rightarrow \mathbb{E}^{\mathrm{m}+1}$ is called a $\mathrm{k}$-type slant helix if the vector field $\mathrm{V}_{\mathrm{k}}(1 \leq \mathrm{k} \leq \mathrm{m}+1)$ makes a constant angle $\theta_{\mathrm{k}}$ with the fixed direction $\overrightarrow{\mathrm{U}}$ in $\mathbb{E}^{\mathrm{m}+1}$, that is

$$
<\overrightarrow{\mathrm{u}}, \mathrm{v}_{\mathrm{k}}>=\cos \theta_{\mathrm{k}}, \theta_{\mathrm{k}} \neq \frac{\pi}{2}
$$

A 1-type slant helix is known as cylindrical helix [2] or generalized helix [13], [4]. For the characterization of generalized helices in $(n+2)$-dimensional Lorentzian space $\mathbb{L}^{n+2}$ see [19].

We give the following result;

Theorem 2 Let $\gamma=\gamma(\mathrm{s})$ be a regular generic curve in $\mathbb{E}^{\mathrm{m}+1}$. If $\mathrm{C}_{\gamma}: \mathrm{s} \rightarrow$ $\mathrm{C}_{\gamma}(\mathrm{s})$ is the focal representation of $\gamma$ then the following statements are valid;

i) If $\gamma$ is a 1-slant helix then the focal representation $\mathrm{C}_{\gamma}$ of $\gamma$ is an $(\mathrm{m}+1)$ slant helix in $\mathbb{E}^{\mathrm{m}+1}$.

ii) If $\gamma$ is an $(\mathrm{m}+1)$-slant helix then the focal representation $\mathrm{C}_{\gamma}$ of $\gamma$ is a 1 -slant helix in $\mathbb{E}^{\mathrm{m}+1}$.

iii) If $\gamma$ is a $k$-slant helix $(2<\mathrm{k}<\mathrm{m})$ then the focal representation $\mathrm{C}_{\gamma}$ of $\gamma$ is an $(\mathrm{m}-\mathrm{k}+2)$-slant helix in $\mathbb{E}^{\mathrm{m}+1}$.

Proof. i) Suppose $\gamma$ is a 1 -slant helix in $\mathbb{E}^{\mathrm{m}+1}$. Then by Definition 3 the vector field $V_{1}$ makes a constant angle $\theta_{1}$ with the fixed direction $\overrightarrow{\mathrm{u}}$ defined in (11), that is

$$
<\overrightarrow{\mathrm{u}}, \mathrm{v}_{1}>=\cos \theta_{1}, \theta_{1} \neq \frac{\pi}{2}
$$

For the focal representation $\mathrm{C}_{\gamma}(\mathrm{s})$ of $\gamma$, we can choose the orthogonal basis

$$
\left\{V_{1}(s)=t, V_{2}(s)=n_{1}, \ldots, V_{m+1}(s)=n_{m}\right\}
$$

such that the equalities (9) or (10) is hold. Hence, we get,

$$
<\overrightarrow{\mathrm{u}}, \mathrm{v}_{1}>=<\overrightarrow{\mathrm{u}}, \mathrm{t}>=<\overrightarrow{\mathrm{u}}, \pm \mathrm{N}_{\mathrm{m}}>=\text { cons. }
$$

where $\left\{T, N_{1}, N_{2}, \ldots, N_{m}\right\}$ is the Frenet frame of $C_{\gamma}$ at point $C_{\gamma}(s)$. From the equality (17) it is easy to see that $C_{\gamma}$ is an $(m+1)$-slant helix of $\mathbb{E}^{m+1}$.

ii) Suppose $\gamma$ is an $(m+1)$-slant helix in $\mathbb{E}^{m+1}$. Then by Definition 3 the vector field $V_{\mathfrak{m}+1}$ makes a constant angle $\theta_{\mathfrak{m}+1}$ with the fixed direction $\vec{u}$ defined in (11), that is

$$
<\overrightarrow{\mathrm{u}}, \mathrm{v}_{\mathrm{m}+1}>=\cos \theta_{\mathrm{m}+1}, \theta_{\mathrm{m}+1} \neq \frac{\pi}{2}
$$


For the focal representation $\mathrm{C}_{\gamma}(\mathrm{s})$ of $\gamma$, one can get

$$
<\overrightarrow{\mathrm{u}}, \mathrm{v}_{\mathrm{m}+1}>=<\overrightarrow{\mathrm{u}}, \mathrm{n}_{\mathrm{m}}>=<\overrightarrow{\mathrm{u}}, \mathrm{T}>=\text { cons. }
$$

where $\left\{V_{1}=t, V_{2}=n_{1}, \ldots, V_{m+1}=n_{m}\right\}$ and $\left\{T, N_{1}, N_{2}, \ldots, N_{m}\right\}$ are the Frenet frame of $\gamma$ and $C_{\gamma}$, respectively. From the equality (19) it is easy to see that $\mathrm{C}_{\gamma}$ is a 1-slant helix of $\mathbb{E}^{\mathrm{m}+1}$.

iii) Suppose $\gamma$ is a $k$-slant helix in $\mathbb{E}^{m+1}(2 \leq k \leq m)$. Then by Definition 3 the vector field $V_{k}$ makes a constant angle $\theta_{k}$ with the fixed direction $\vec{u}$ defined in (11), that is

$$
<\overrightarrow{\mathrm{u}}, \mathrm{v}_{\mathrm{k}}>=\cos \theta_{\mathrm{k}}, \theta_{\mathrm{k}} \neq \frac{\pi}{2}, 2 \leq \mathrm{k} \leq \mathrm{m}
$$

Let $C_{\gamma}(s)$ be the focal representation of $\gamma$. Then using the equalities (9) or (10) we get

$$
<\overrightarrow{\mathrm{u}}, \mathrm{v}_{\mathrm{k}}>=<\overrightarrow{\mathrm{u}}, \mathrm{n}_{\mathrm{k}-1}>=<\overrightarrow{\mathrm{U}}, \mathrm{N}_{\mathrm{m}-\mathrm{k}+1}>=\text { cons., } 2 \leq \mathrm{k} \leq \mathrm{m}
$$

where

$$
\left\{V_{1}=t, V_{2}=n_{1}, \ldots, V_{m+1}=n_{m}\right\}
$$

and

$$
\left\{\widetilde{V}_{1}=\mathrm{T}, \widetilde{\mathrm{V}}_{2}=\mathrm{N}_{1}, \ldots, \widetilde{\mathrm{V}}_{\mathrm{m}-\mathrm{k}+2}=\mathrm{N}_{\mathrm{m}-\mathrm{k}+1}, \ldots, \widetilde{\mathrm{V}}_{\mathrm{m}+1}=\mathrm{N}_{\mathrm{m}}\right\}
$$

are the Frenet frame of $\gamma$ and $C_{\gamma}$, respectively. From the equality (21) it is easy to see that $C_{\gamma}$ is an $(m-k+2)$-slant helix of $\mathbb{E}^{m+1}$.

\section{References}

[1] A. T. Ali, Inclined curves in the Euclidean 5 -space $\mathbb{E}^{5}, J$. Adv. Res. Pure Math., 1 (1) (2009), 15-22.

[2] A. T. Ali, R. Lopez, Some characterizations of inclined curves in Euclidean $\mathbb{E}^{\mathfrak{n}}$ space, Novi Sad J. Math., 40 (1) (2010), 9-17.

[3] A. T. Ali, M. Turgut, Some characterizations of slant helices in the Euclidean space $\mathbb{E}^{n}$, Hacet. J. Math. Stat., 39 (3) (2010), 327-336.

[4] M. Barros, General helices and a theorem of Lancert, Proc. Amer. Math. Soc., 125 (1997), 1503-1509. 
[5] B. Y. Chen, Total mean curvature and submanifolds of finite type, World Sci., Singapore, 1984.

[6] B. Y. Chen, J. Deprez, P. Verheyen, Immersions with geodesics of 2-type, Geom. and topol. of submanifolds IV, Belgium, 1992.

[7] C. Camcı, K. Ilarslan, L. Kula, H. H. Hacısalihoğlu, Harmonic curvatures and generalized helices in $\mathbb{E}^{n}$, Chaos Solitons Fractals, 40 (2009), 25902596.

[8] H. Gluck, Higher curvatures of curves in Euclidean space, Amer. Math. Monthly, 73 (1966), 699-704.

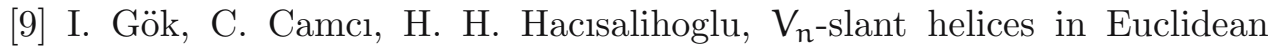
n-space $\mathbb{E}^{\mathrm{n}}$, Math. Commun., 14 (2009), 317-329.

[10] H. A. Hayden, On a generalized helix in a Riemannian n-space, Proc. Lond. Math. Soc., 32 (1931), 37-45.

[11] S. Izumiya, N. Takeuchi, New special curves and developable surfaces, Turkish J. Math., 28 (2) (2004), 531-537.

[12] F. Klein, S. Lie, Uber diejenigen ebenenen kurven welche durch ein geschlossenes system von einfach unendlich vielen vartauschbaren linearen Transformationen in sich übergehen, Math. Ann., 4 (1871), 50-84.

[13] M. C. Romero-Fuster, E. Sanabria-Codesal, Generalized helices, twistings and flattenings of curves in n-space, Mat. Contemp., 17 (1999), 267-280.

[14] J. Monterde, Curves with constant curvature ratios, Bull. Mexican Math. Soc. Ser. 3A, 13 (1) (2007), 177-186.

[15] G. Öztürk, K. Arslan, H. H. Hacisalihoglu, A characterization of ccrcurves in $\mathbb{R}^{\mathrm{m}}$, Proc. Est. Acad. Sci., 57 (4) (2008), 217-2248.

[16] R. Uribe-Vargas, On Vertices, focal curvatures and differential geometry of space curves, Bull. Braz. Math. Soc. (N.S.), 36 (2005), 285-307.

[17] R. Uribe-Vargas, On singularities, 'perestroikas' and differential geometry of space curves, L'Enseigement Math., 50 (2004), 69-101. 
[18] R. Uribe-Vargas, Singularites symplectiques et de contact en Geometrie differentielle des courbes et des surfaces, PhD. Thesis, Universite Paris 7, 2001.

[19] A. F. Yalınız, H. H. Hacısalihoğlu, Null generalized helices in $\mathbb{L}^{n+2}$, Bull. Malays. Math. Sci. Soc., 30(1) (2007), 74-85.

Received: October 22, 2014 